\title{
$F P_{\infty}$ GROUPS AND HNN EXTENSIONS ${ }^{1}$
}

\author{
BY KENNETH S. BROWN AND ROSS GEOGHEGAN
}

A group $G$ is said to be of type $F P_{\infty}$ if the $\mathbf{Z} G$-module $\mathbf{Z}$ admits a projective resolution $\left(P_{i}\right)$ of finite type (i.e., with each $P_{i}$ finitely generated). If $G$ is finitely presented, this is equivalent by Wall $[5,6]$ to the existence of an Eilenberg-Mac Lane complex $K(G, 1)$ of finite type (i.e., with finitely many cells in every dimension). Up to now, all known torsion-free groups of type $F P_{\infty}$ have had finite cohomological dimension; in fact, they have admitted a finite $K(G, 1)$-complex. We announce here the first known example of a torsion-free group of type $F P_{\infty}$ with infinite cohomological dimension. This solves Wall's problem F11 [7]. We show in addition that our group, which we denote by $F$, satisfies $H^{n}(F, \mathbf{Z} F)=0$ for all $n$. As far as we know, $F$ is also the first example of an $F P_{\infty}$ group with this property. The vanishing of $H^{*}(F, \mathbf{Z} F)$ is a consequence of results of independent interest concerning the cohomology of HNN extensions (or, more generally, fundamental groups of graphs of groups) with free coefficients.

The group $F$ is defined by the presentation $\left\langle x_{0}, x_{1}, x_{2}, \ldots ; x_{i}^{-1} x_{n} x_{i}=x_{n+1}\right.$ for $i\langle n\rangle$. It has previously arisen in two contexts: (i) finitely presented infinite simple groups (R. J. Thompson [unpublished]); and (ii) unsplittable free-homotopy idempotents (Freyd and Heller [3], Dydak and Minc [2]). F was previously known to be finitely presented, torsion-free, and of infinite cohomological dimension. (In fact, $F$ has a subgroup which is free abelian of infinite rank.) Our contribution, therefore, is

TheOREM 1. The group $F$ described above is of type $F P_{\infty}$ and satisfies $H^{*}(F, \mathbf{Z} F)=0$.

The proof that $F$ is of type $F P_{\infty}$ goes as follows. Let $\phi: F \rightarrow F$ be the shift map, $\phi\left(x_{i}\right)=x_{i+1}$. Note that $\phi^{2}=T_{x_{0}} \circ \phi$, where $T_{x_{0}}$ is the conjugation map $x \mapsto x_{0}^{-1} x x_{0}$; thus $\phi$ is idempotent up to conjugacy. We construct the universal example of a semicubical complex $K$ with (a) a free right $F$-action; (b) a basepoint-preserving cubical endomorphism $\psi: K \rightarrow K$ compatible with $\phi$; and (c) a homotopy from $\psi^{2}$ to $\rho_{x_{0}} \circ \psi$ compatible with $\phi^{2}$, where $\rho_{x_{0}}(e)=$ $e x_{0}$. (The motivation for this comes from (ii) above; $K$ should be thought of as the universal cover of a complex with a free-homotopy idempotent, and $\psi$ should be thought of as a lift of the idempotent to $K$.) We prove by a direct combinatorial argument that $K$ is acyclic; the chain complex $C$ of $K$ is therefore a free resolution of $\mathbf{Z}$ over $\mathbf{Z F}$. Unfortunately, $C$ is not of finite type. But we are able to find a contractible chain subcomplex $D \subset C$ such

Received by the editors April 19, 1983.

1980 Mathematics Subject Classification. Primary 20J05; Secondary 20E06, 18G20, 55P20.

${ }^{1}$ Authors partially supported by the National Science Foundation. 
that the quotient $P=C / D$ is free of finite rank in every dimension. This is then the desired finite type resolution.

One can give a direct description of this resolution $P$. It is free of rank 1 in dimension 0 and free of rank 2 in every positive dimension. Moreover, there are formulas for computing the boundary operator inductively. In spite of this explicit description of $P$, however, we know of no proof of its acyclicity other than the one outlined above which uses the "big" resolution $C$.

We turn now to the assertion in Theorem 1 that $H^{*}(F, \mathbf{Z} F)=0$. Let $F_{1}$ be the subgroup of $F$ generated by the $x_{i}$ for $i \geq 1$. It is known that $F_{1}$ is isomorphic to $F$ via the shift map $\phi$ and that $F$ is the HNN extension of $F_{1}$ with respect to the monomorphism $\phi \mid F_{1}$ (with $x_{0}$ as the stable letter). We now appeal to a general result about HNN extensions in which the base group and associated subgroups satisfy appropriate finiteness conditions; for simplicity, we will state a special case of this result which suffices for the present application.

THEOREM 2. Let $G$ be an $H N N$ extension in which the base group $G_{1}$ and associated subgroups $A$ and $B$ are of type $F P_{\infty}$. Assume that one of the associated subgroups, say $A$, has the property that the restriction map $H^{*}\left(G_{1}, \mathbf{Z} G_{1}\right) \rightarrow H^{*}\left(A, \mathbf{Z} G_{1}\right)$ is a monomorphism. Then in the Mayer-Vietoris sequence

$$
\cdots \rightarrow H^{q}(G, \mathbf{Z} G) \rightarrow H^{q}\left(G_{1}, \mathbf{Z} G\right) \stackrel{\alpha}{\rightarrow} H^{q}(A, \mathbf{Z} G) \rightarrow \cdots
$$

the map $\alpha$ is a monomorphism.

This generalizes a result of Bieri [1, Theorem 6.6], in which $A$ and $B$ were both assumed to be of finite index in $G_{1}$. Note that our hypothesis about the restriction map holds whenever one of these subgroups is of finite index. In particular, it holds when $G_{1}=A$, which is the case in our present application with $G=F$ and $G_{1}=A=F_{1}$. If we now assume inductively that $H^{q-1}(F, \mathbf{Z} F)=0$, it follows that $H^{q-1}(F, L)=0$ for any free $\mathbf{Z} F$-module $L$. Since $F_{1} \approx F$, this yields $H^{q-1}\left(F_{1}, \mathbf{Z} F\right)=0$, so the Mayer-Vietoris sequence takes the form

$$
0 \rightarrow H^{q}(F, \mathbf{Z} F) \rightarrow H^{q}\left(F_{1}, \mathbf{Z} F\right) \stackrel{\alpha}{\rightarrow} H^{q}\left(F_{1}, \mathbf{Z} F\right) \rightarrow \cdots
$$

Theorem 2 now implies that $H^{q}(F, \mathbf{Z} F)=0$, as required. This completes the sketch of the proof of Theorem 1 .

To prove Theorem 2, one can give a normal form argument. Alternatively, there is a proof which makes use of the tree associated to the HNN extension [4]. This second proof is of interest because it leads to a generalization of Theorem 2 to fundamental groups of graphs of groups, as follows.

Let $G$ be the fundamental group of a finite graph of groups [4]. We will assume for simplicity that the vertex and edge groups are all of type $F P_{\infty}$, although this hypothesis can be weakened. Let $X$ be the associated tree. For each integer $q$ there is a "coefficient system" $D^{q}$ on $X$ which associates to each vertex or edge $\sigma$ of $X$ the group $H^{q}\left(G_{\sigma}, \mathbf{Z} G_{\sigma}\right)$, where $G_{\sigma}$ is the isotropy subgroup of $G$ at $\sigma$, and which associates to each incidence relation " $v$ is a 


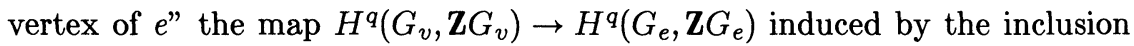
$G_{e} \hookrightarrow G_{v}$ and the canonical projection $\mathbf{Z} G_{v} \rightarrow \mathbf{Z} G_{e}$. Our hypotheses imply that this coefficient system is locally finite in a suitable sense, so that we can form the complex $C_{c}^{*}\left(X, D^{q}\right)$ of cochains with compact supports and hence the cohomology groups $H_{c}^{*}\left(X, D^{q}\right)$. One now verifies that in the Mayer-Vietoris sequence with $\mathbf{Z} G$-coefficients, the map analogous to the map $\alpha$ of Theorem 2 can be identified with the coboundary map $C_{c}^{0}\left(X, D^{q}\right) \rightarrow C_{c}^{1}\left(X, D^{q}\right)$. The content of Theorem 2, then, is that (under the hypotheses of the latter) $H_{c}^{0}\left(X, D^{q}\right)=0$. It is not hard to verify this by directly checking definitions. In the general case this discussion yields

TheOREM 3. Let $G$ be the fundamental group of a finite graph of groups of type $F P_{\infty}$ as above. Then there is a short exact sequence

$$
0 \rightarrow H_{c}^{1}\left(X, D^{q-1}\right) \rightarrow H^{q}(G, \mathbf{Z} G) \rightarrow H_{c}^{0}\left(X, D^{q}\right) \rightarrow 0 .
$$

In particular, this shows that $H^{q}(G, \mathbf{Z} G) \approx H_{c}^{1}\left(X, D^{q-1}\right)$ under the hypotheses of Theorem 2. We will give further generalizations and applications of Theorem 3 elsewhere.

\section{REFERENCES}

1. R. Bieri, Homological dimension of discrete groups, Queen Mary College Math. Notes, London, 1976.

2. J. Dydak, A simple proof that pointed connected FANR-spaces are regular fundamental retracts of ANR's, Bull. Acad. Polon. Sci. Sér. Sci. Math. Astronom. Phys. 25 (1977), 55-62.

3. P. Freyd and A. Heller, Splitting homotopy idempotents. II, unpublished manuscript, 1979.

4. J-P. Serre, Trees, Springer-Verlag, Berlin-Heidelberg-New York, 1980. (Translation of: Arbres, amalgames, $S L_{2}$, Astérisque 46 (1977).)

5. C. T. C. Wall, Finiteness conditions for CW-complexes, Ann. of Math. (2) 81 (1965), 56-69.

6. _ Finiteness conditions for $C W$-complexes. II, Proc. Roy. Soc. A 295 (1966), 129-139.

7. C. T. C. Wall (ed.), List of problems, Homological Group Theory, London Math. Soc. Lecture Notes, No. 36, Cambridge Univ. Press, Cambridge, 1979, pp. 369-394.

Department of Mathematics, Cornell University, Ithaca, New York 14853

Department of Mathematical Sciences, State University of New York, Binghamton, New York 13901 
